$\begin{array}{ll}\text { Research Square } & \begin{array}{l}\text { Preprints are preliminary reports that have not undergone peer review. } \\ \text { They should not be considered conclusive, used to inform clinical practice, } \\ \text { or referenced by the media as validated information. }\end{array}\end{array}$

\title{
A guide for Physiotherapeutic assistance during pregnancy, labour, and the postpartum period in times of COVID-19
}

Ana Carolina Rodarti Pitangui

Universidade de Pernambuco

Patricia Driusso

Federal University of São Carlos

Lilian Rose Mascarenhas

Federal University of Para

Marcela Ponzio Pinto Silva

State University of Campinas

Mariana Maia de Oliveira Sunemi

State University of Campinas

Claudia de Oliveira

Universidade Santa Cecília

Rubneide Barreto Silva Gallo

Federal University of Paraná

Cristine Homsi Jorge Ferreira ( $\nabla$ cristine@fmrp.usp.br)

Sao Paulo State University

Research Article

Keywords: COVID-19, Pandemic, Women's Health, Physiotherapy, Rehabilitation, Obstetrics, Labour

Posted Date: February 12th, 2021

DOI: https://doi.org/10.21203/rs.3.rs-154738/v1

License: @ (i) This work is licensed under a Creative Commons Attribution 4.0 International License. Read Full License 


\section{Abstract \\ Background}

Many recommendations contained in the guidelines available to date are specifically related to clinical practice in obstetrics. Although many physiotherapists are facing similar issues to gynecologists and obstetricians about the care and well-being of pregnant women with confirmed or suspected COVID-19, no specific guidelines were found regarding the physiotherapist's practice in this area. This article aimed to offer guidance regarding physiotherapy in obstetrics during the COVID-19 pandemic.

\section{Methods}

A group of experts in physiotherapy in women's health performed a literature search and proposed a clinical guideline for the physiotherapy management of assistance during pregnancy, labour, and the postpartum period in times of COVID-19. This document was reviewed by other physiotherapists, which analyzed the suggested topics and reached a consensus.

\section{Results}

The recommendation addresses in general terms concerning pregnancy and the postpartum period during the COVID-19 pandemic, summarize the physiotherapy care in obstetrics, the practice of the physiotherapist at the outpatient level, physiotherapy clinics, and at pregnant women's home.

Recommendations on the safety of the care environment; general aspects of care to pregnant women without symptoms of COVID-19; specific aspects of care for pregnant women without symptoms of COVID-19; care to pregnant women with COVID-19; the practice of activity and physical exercises monitored in pregnant women and the postpartum in times of pandemic and about the physiotherapist's assistance in the maternity hospital with parturients and puerperal women are still presented.

\section{Conclusion}

This study provides some guidance for continuity of the physiotherapist's work in obstetrics during the COVID-19 pandemic. Telephysiotherapy is a possibility for the permanence of care during the pandemic, opening new perspectives for the expansion of practice and research in women's health, especially in obstetrics.

\section{Background}

On March 11, 2020, the World Health Organization (WHO) declared a pandemic, due to COVID-19. Health systems and professionals around the world are under increasing pressure concerning the appropriate approach to ensure good quality health at different levels of health care during pregnancy, childbirth, and postpartum, making this issue a priority related to public health. The implications of this disease for pregnancy, childbirth, and postpartum are still uncertain [1].

Pregnancy is a period in which the female body undergoes many hormonal and anatomical changes adaptations lead to numerous musculoskeletal symptoms that can considerably affect the quality of life and functionality of women [2]. Physical inactivity and excessive weight gain in pregnancy, have been documented as independent risk factors for maternal obesity and related pregnancy complications, including gestational diabetes mellitus [3]. Numerous benefits can be obtained through the physical therapy interventions used in this phase of the female life cycle [4-15].

It should be highlighted the importance of physical exercises monitored to maintain physical fitness, prevent metabolic problems that may increase the risk of complications in pregnancy in addition to COVID-19, with emphasis on overweight and obesity [16]. Women who exercise during pregnancy have presented benefits such as decreased cesarean birth and operative vaginal delivery [17]. Physical activity also can be an important factor in the prevention of depressive disorders in women in the postpartum period [18]. Pregnant women with COVID-19 are considered to be at high risk and non-serious cases in social isolation will require physiotherapy guidelines to improve comfort and reduce complications related to immobility, preferably through the call center.

The interruption of the physical therapist's assistance in the maternity hospital in women with obstetrics complications can have negative repercussions related to the various systems, especially respiratory, circulatory, and motor complications favored by the restriction to the bed. Also, it is believed that there will be a worsening in the quality of life of women, which overlaps the impact that social distance can have on raising levels of stress and anxiety [19].

Physiotherapeutic care for women in labour provides pain relief and improves the progression in labor, decreasing the use of analgesic medication and unnecessary medical interventions contributing to women's autonomy and a positive experience [7-11].

In the immediate postpartum period, physiotherapy assistance can improve women's global functionality, posture, facilitating breastfeeding, preventing circulatory, respiratory, and complications. There is evidence for the use of physiotherapeutic interventions to provide pain relief related to cesarean section [12], perineal trauma [13-15] and, assist in the process and demands related to breastfeeding [20, 21]. Additionally, in the postpartum period, the musculoskeletal rehabilitation process should also be started early in the maternity hospital environments.

Many recommendations contained in the guidelines available to date are specifically related to clinical practice in obstetrics. Although many physiotherapists are facing similar issues to gynecologists and obstetricians about the care and well-being of pregnant women with confirmed or suspected COVID-19, no 
specific guidelines were found regarding the physiotherapist's practice in this area. Therefore, this manuscript aims to offer guidance regarding physiotherapy care in obstetrics during pandemic times.

\section{Methods}

\section{Study design}

This is a descriptive study proposing guidance and recommendations based on a literature review and a group of expert's opinions about the topic.

\section{Development of the recommendations and consensus approach}

A group of experts that are members of the Brazilian Association of Physiotherapy in Women's Health (ABRAFISM), came together to rapidly prepare a clinical recommendation for physiotherapy management to pregnant women, women during childbirth and, postpartum women in times of COVID-19.

A literature review was conducted from inception to June 092020 in the following databases: Pubmed, PEDro, Web of Science collection and, Embase. All searches were restricted to English and Portuguese languages. For all topics, we sought existing systematic reviews and clinical trial studies. We have also consulted the guidelines of international associations and entities, such as the World Confederation for Physical Therapy (WCPT), WHO, American College of Obstetricians and Gynecologists (CCOG), Royal College of Obstetricians and Gynaecologists (RCOG), Brazilian Federation of Gynecology and Obstetrics Associations (FEBRASGO), Brazilian Ministry of Health (MS) and Cochrane Pregnancy and Childbirth.

The terms used in the search strategy in all database were: ("physiotherapy" OR "physical therapy" OR "rehabilitation") AND ("pregnant" OR "pregnancy" OR "pregnant women" OR "antenatal woman" OR "postnatal women" OR "childbirth" OR "labor" OR "delivery" OR "vaginal delivery" OR "cesarean section" OR "birth" OR "puerperium" OR "postpartum" OR "episiotomy" OR "lumbopelvic pain" OR "urinary incontinence" OR "exercise" OR "pelvic floor" OR incontinence OR "labour pain" OR "birth ball" OR "non-pharmacological” OR "transcutaneous electrical nerve stimulation" "massage" OR "painful nipples" OR "breastfeeding" OR "lactation" OR "mobile app" OR "digital health" OR telemedicine OR "tele rehabilitation" OR telehealth OR "telephone-delivered care" OR "telephone health" OR "home treatment” OR "home-based” OR “COVID-19" OR “COVID 19” OR “COVID” OR "coronavirus").

It was decided to develop consensus recommendations. Two independent reviewers (ACRP) and (CHJF) performed the selection process of studies through titles, abstracts, and full-text reading based on the previously described proposed inclusion criteria. The reference lists of included studies were screened independently to identify possible studies not retrieved by the electronic search. Any disagreements during the selection process were resolved discussion until a consensus was reached by the group of specialists. On 23 June 2020, the lead author distributed draft recommendations to all authors. The lead author organized all comments for further discussion in the group. Each recommendation was discussed until a consensus was reached and they were, therefore, grouped according to their similarities and allocated into categories.

Subsequently, the working group approved the recommendations.

Twenty-one people participated in the development process. The recommendations were distributed to all the Physiotherapists members of the ABRAFISM on the website on 04 June 2020.

\section{What are the risks of the COVID-19 to pregnant women?}

The hormonal state and the reduced chest expansion increase pregnant women's risk of respiratory infections [22]. Additionally, data concerning the COVID-19 epidemic, demonstrated that pregnant women and their neonates are exposed to a higher risk of poor outcomes [23]. Little is known about the impact of COVID-19 on pregnancies, perinatal, and neonatal outcomes [24]. The risk of being admitted to intensive care units (ICU) may be higher in pregnant and postpartum women with laboratory-confirmed SARS-CoV-2, compared with non-pregnant women of similar age. Pregnant women may be more susceptible to COVID-19 complications, but with adequate and timely intensive care the survival rate could be similar to non-pregnant women [25].

Normally, pregnant women with COVID-19 presented with mild symptoms, mostly fever and cough, a considerable number of patients stayed asymptomatic, and only a few cases developed dyspnea requiring oxygen therapy or admission in ICU for intensive treatment. It is feasible to hypothesize that the worse neonatal outcome was related to the poor clinical conditions of the mother during pregnancy and delivery, rather than being caused by vertical transmission of the infection. However, until now, data are limited [24].

The data from Brazil, Iran, and Mexico, raising the possibility of an increased risk of maternal death from COVID-19. The high birth rates and limited resources for healthcare provision in Brazil will uncover the increased risk for maternal death because of COVID-19 and highlight the need for corrective measures for adequate prenatal and postnatal care. It was hypothesized that higher birth rates, worse population health status, and poor quality of obstetrics care, caused by the management of the pandemic, would cause a rise in the number of deaths and also the case fatality rate [26, 27].

Pregnant patients with comorbidities such as obesity and gestational diabetes may be at an even higher risk for severe illness consistent with the general population with similar comorbidities [29]. The other important factor is the highest rate of cesarean sections, which increased the risk of postoperative morbidity and mortality for patients with COVID-19 undergoing surgery [28].

The COVID-19 can cause serious adverse outcomes during pregnancy, such as fetal distress and preterm delivery [30]. It is not clear that these outcomes were related to maternal infection, and at this time the risk of adverse infant outcomes is not known. There is limited evidence about the vertical transmission, prevalence and, clinical features of COVID-19 during pregnancy, birth, and the postnatal period. [31]. The general recommendation concerning pregnancy and the postpartum period during the COVID-19 pandemic are present in Box 1. 


\section{Physiotherapeutic assistance in pregnant, parturient, and postpartum women during the pandemic.}

Face-to-face care should be avoided to the maximum during the COVID-19 pandemic. Modifying the care approach is appropriate when the risk of exposure to COVID-19 is high and health resources for outpatient care are reduced. A pre-appointment phone screening should be done before any essential face-to-face consultation to verify the existence of comorbidities or COVID-19 symptoms.

Regarding the presence of the physiotherapist in the maternity hospital teams, due to the risk of exposure to COVID-19 by the health team and patients, it may be necessary to modify the policies according to the specificities and norms of each institution. However, the WHO emphasizes that all women have the right to have a positive and safe experience in labor, regardless of the status of COVID-19. Therefore, continuing to follow the precepts of the humanization of care is essential. According to the WHO, strategies for pain relief and mobility during labor should be maintained [36].

Work-related care in only one health institution should also be adopted whenever possible, as well as the organization of the schedule of the physiotherapists, exclusively in the sectors destined to attend in labor and postpartum women with or without COVID-19. Box 2 presents a summary of physiotherapy care in obstetrics and the recommendations regarding personal protective equipment (PPE).

\section{Outpatient clinics, clinics, and homes}

The recommendations regarding the practice of the physiotherapist at the outpatient level, physiotherapy clinics and, at pregnant women's home were classified and presented: the safety of the care environment [Box 3]; general aspects of care to pregnant women without symptoms of COVID-19 [Box 4]; specific aspects of care to pregnant women without symptoms of COVID-19 [Box 5]; care to pregnant women with COVID-19 [Box 6] and the practice of physical exercise in pregnant and postpartum women during the pandemic [Box 7].

Recommendations related to specific physiotherapy guidelines for pregnant women with COVID-19 should be interpreted with caution since there are no specific studies. These recommendations were based on the opinion of a group of experts.

\section{The assistance of the physiotherapist in maternity hospitals}

In some countries, the physiotherapist works in maternity hospitals assessing women in labor to prescribe, apply, and guide, the use of physiotherapeutic resources for pain relief and the progression of labor. The work of the physiotherapist involves also a specific knowledge about the body and pelvis biomechanics in labor and delivery, respecting the time and preferences of each woman in the process of labor, and the global aspects of her health and evolution in labor [8-10]. The role of the physiotherapist encompasses the immediate postpartum period, seeking to prevent deep vein thrombosis, to improve the respiratory, cardiac, gastrointestinal, locomotor, postural, urinary functions, among others, encouraging the bond between mother and the newborn. Also, it seeks to relieve pain related to perineal trauma and cesarean section favoring the healing processes [12-15].

The instructions related to the late and remote postpartum are often initiated by the physiotherapist during the period when the postpartum women are still hospitalized. The physiotherapists aim to familiarize the postpartum woman with the rehabilitation process to help in the return of her body to the pregestational condition and optimizing its functionality, treating the discomforts and dysfunctions that may have persisted or arisen, due to pregnancy and childbirth. The above recommendations in general are also applicable to outpatient care in maternity hospitals.

Studies have shown beneficial effects of early prone positioning for acute respiratory distress syndrome, including decreased mortality. Though, pregnant women were excluded from the trials. A current report has also found that prone positioning to be particularly helpful for patients with CoVID-19 with the moderate or severe respiratory disease [41]. However, data are limited, case reports and expert experience recommend that pregnant women may be safely prone and that prone positioning may be particularly beneficial in these patients owing to its ability to relieve both diaphragmatic compression from abdominal contents and aortocaval compression from the gravid uterus if performed correctly [42].

A randomized, controlled trial analyzed the influence of lying in a prone position on a specially designed stretcher on the maternal-fetal hemodynamic parameters and comfort of pregnant women. The authors created a special prototype stretcher for pregnant women. They concluded that the prone position was considered safe and comfortable and could be advantageous for improving oxygen saturation and reducing the systolic blood pressure and respiratory rate [43].

Prone positioning during pregnancy requires particular considerations. Routine indications and contraindications apply, with additional caution for patients within 2 days postoperative from cesarean delivery owing to concerns for incisional complications and pain in the immediate postoperative period. Prone positioning for patients at 34 weeks of gestation or more may be more technically difficult owing to the large gravid uterus at advanced gestational ages, and the risks and benefits of delivery before prone positioning should be considered [41]. More recommendations about the physiotherapist's assistance in the maternity hospital with parturients and postpartum women can be seen in Box 8 .

\section{Discussion}

Although physiotherapy is considered an essential activity for many centuries, face-to-face care should be restricted to the maximum in times of COVID-19 pandemic. The possibility of a telephone contact should be taken into account before the face-to-face and essential physiotherapy consultation to check for the existence of COVID-19 comorbidities or symptoms. Use tele-triage methods for assessing and caring for all patients to reduce the volume of persons seeking care in services, in particular during times of high transmission of COVID-19 [37].

However, locoregional epidemiological issues and the need for contact restriction should be taken into account as well as indications for physiotherapy care, availability of human resources, and adequate physical space to a safe attendance for patients and the health team providers. Digital practice will not be the 
solution to all the challenges that physiotherapists are facing, especially in countries where internet access unfeasible for many patients and physiotherapists [39].

The advantages of telephysiotherapy including encouragement of self-management, increases in flexibility for healthcare delivery and decreases in sick-leave duration, reduced costs to transport patients and physiotherapists to health services, time saved for patients waiting to be treated and, being able to assist patients from distant regions. It is a chance for physiotherapists to work innovatively using technology, valuing the evaluation of characteristics of the patients' history and habits, communication skills and, provision of instructions to help them change life habits and adhere to treatment [39, 40].

Telephysiotherapy should prioritize aspects of patients' self-reports, preferably using simplified validated questionnaires. Some specific aspects of the distance physical examination involving the musculoskeletal system have good reliability, and detailed explanations can be offered to patients, including postural and self-assessment palpation of the musculoskeletal system [40].

Exposure of the naked genitalia of patients and vaginal or anal self-palpation are not recommended for self-examination of pelvic floor muscle function during video calls or through means that can expose the patient and embarrass her. Remote physical examination findings should be interpreted with caution. Digital physical therapy may not be the ideal tool for a complete assessment of the pregnant woman, but it may be the only resource in some situations, its limitations must be explained to the patient. It is indicated for routine prenatal or postpartum physiotherapeutic care, prescription and, exercise supervision for pregnant women in good general health, good eyesight, and hearing.

The sessions should offer quality care and may include postural, ergonomic guidelines, exercises for physical conditioning, general kinesiotherapy, pelvic floor muscle training and, other muscle groups. It is recommended to continue offering educational activities digitally, including preparation for childbirth and the postpartum period. The educational materials should be organized by the professional and shared virtually (e-mail or digital platforms) with the patients (leaflets, booklets, anatomical teaching materials) preferably before the service sessions. Clear and empathetic communication with the patient through digital media and maximizing self-care guidelines and lifestyle changes are very important and feasible to be offered using telephysiotherapy. All appointments must be duly registered in the patients' medical records and consent must be obtained for online appointments.

Face-to-face and individualized care should be considered in cases of urgency in face-to-face assessment for the use of physical therapy techniques for pain relief in which physical therapy resources are not viable digitally and cannot be replaced by another remote medium that has the same clinical effect.

Regarding the presence of the physiotherapist in the maternity team, due to the risk of exposure to COVID-19 by the health team and patients, it may be necessary to modify the policies according to the specificities and rules of each institution. However, WHO emphasizes that all women should have a positive and safe experience in labor, regardless of the status of COVID-19 [36].

Therefore, continuing to follow the precepts the humanization of assistance is essential. According to the WHO, the provision of strategies for pain relief and mobility during labor should be maintained. They recommend that non-pharmacological resources for pain relief in labor continue to be offered [36]. In some countries, the physiotherapist provides direct assistance to women in labor while in others this professional offers important support to the team involved in labor assistance.

It is important to highlight that the work of the physiotherapist should be maintained whenever possible since it provides the effective use of nonpharmacological analgesia and specific physiotherapeutic resources to parturients [8-11] and postpartum [13-15, 20, 21] women fundamental to the humanization of care.

In some maternities that do not have a number adequate number of contracted physiotherapists, it will be necessary to reassign the professionals' scales to maintain the face-to-face and essential assistance, or at least the assistance provided by the same to the team, avoiding excessive exchanges during the working day and maintaining security in the assistance. Care related to work in multiple health institutions should also be adopted whenever possible, as well as the organization of the scale of contracted physiotherapists, divided exclusively into contracted physiotherapists, divided sectors that care for parturients/mothers with or without COVID-19.

The physiotherapist's work with labor and postpartum women in maternity wards should be maintained whenever possible, considering all locoregional epidemiological issues and following the current rules of safety and use of personal protective equipment (PPE) by the physiotherapist and wearing a mask by part of the patient.

There are some limitations we must acknowledge, the first one is the short period we conducted the literature search and the development process of the recommendations. Due to the recent occurrence of COVID-19, the clinical guidelines contained in this recommendation may change as new articles and knowledge about the disease are emerging. The recommendations were compiled based on the best available evidence but mostly based on expert's opinions, combined with no or low levels of evidence. Though, it was feasible to offer some directions to professionals working in the area. However, to date, no specific articles and guidelines have been found on the role of physiotherapy in women's health with a focus on obstetrics and the COVID-19.

Telephysiotherapy will not be a viable alternative for all patients, but it offers prospects for the continuity of care to some patients [40]. Maintaining continuity of care to the extent possible can avoid additional negative consequences from delayed preventive, chronic, or routine care [37].

\section{Conclusions}

This article presented some guidance related to physiotherapeutic assistance during pregnancy, labour, and the postpartum period in times of COVID-19. Though, local epidemiological situations and sustained declines in the number of COVID-19 cases must be taken into account for a safe return of face-to-face 
care. Telephysiotherapy is a possibility for the permanence of care during the pandemic, opening new perspectives for the expansion of practice and research in women's health, especially in obstetrics.

\section{Declarations}

\section{Acknowledgments}

We are grateful to ABRAFISM, and the advisory and drafting committee for recommendations for physiotherapeutic assistance during pregnancy, labour, and the postpartum period in times of COVID-19 for collaboration and support.

\section{Authors' contributions}

ACRP was responsible for drafting the manuscript, coordinated, interpret the data, and approved the final manuscript as submitted. CHJF, coordinated and supervised data, and critically reviewed the manuscript. LRM, coordinated and supervised data. MPPS, C de O, MM de OS, RBSG, and, PD critically reviewed the manuscript. The authors approved the final manuscript as submitted and agree to be accountable for all aspects of the work.

\section{Consent for publication}

Not applicable.

\section{Competing interests}

The authors have no potential conflicts of interest to disclose.

\section{Ethics approval and consent to participate}

Not applicable' for that section.

\section{Availability of data and materials}

Not applicable' for that section.

\section{Funding}

We thank the University of São Paulo for the financial assistance to publish.

\section{References}

1. Cochrane Pregnancy and Childbirth. COVID-19 review of national clinical practice guidelines for key questions relating to the care of pregnant women and their babies. Available at: https://pregnancy.cochrane.org/news/covid-19-review-national-clinical-practice-guidelines-key-questions-relating-care-pregnant. Accessed Oct. 18, 2020.

2. Ireland ML, Ott SM. The effects of pregnancy on the musculoskeletal system. Clin Orthop Relat Res. 2000 Mar;(372):169-79.

3. Artal R. The role of exercise in reducing the risks of gestational diabetes mellitus in obese women. Best Pract Res Clin Obstet Gynaecol 2015;29:123-3.

4. Miquelutti MA, Cecatti JG, Makuch Evaluation of a birth preparation program on lumbopelvic pain, urinary incontinence, anxiety and exercise: a randomized controlled trial. BMC Pregnancy Childbirth. 2013;13:154.

5. Woodley SJ, Lawrenson P, Boyle R, et al. Pelvic floor muscle training for preventing and treating urinary and faecal incontinence in antenatal and postnatal women. Cochrane Database Syst Rev. 2020;5(5):CD007471.

6. Miquelutti MA, Cecatti JG, Makuch MY. Developing strategies to be added to the protocol for antenatal care: an exercise and birth preparation program. Clinics (Sao Paulo). 2015;70(4):231-236.

7. Leung RW, Li JF, Leung MK, et al. Efficacy of birth ball exercises on labour pain management. Hong Kong Med J. 2013;19(5):393-399.

8. Delgado A, Maia T, Melo RS, Lemos A. Birth ball use for women in labor: A systematic review and meta-analysis. Complement Ther Clin Pract. 2019;35:92101.

9. Smith CA, Levett KM, Collins CT, Jones L. Massage, reflexology and other manual methods for pain management in labour. Cochrane Database Syst Rev. 2012 Feb 15;(2):CD009290.

10. Gallo RBS et al. Sequential application of non-pharmacological interventions reduces the severity of labour pain, delays use of pharmacological analgesia, and improves some obstetric outcomes: a randomised trial. J Physiother. 2018 Jan;64(1):33-40.

11. Santana LS et al. Transcutaneous electrical nerve stimulation (TENS) reduces pain and postpones the need for pharmacological analgesia during labour: a randomised trial. J Physiother. 2016 Jan;62(1):29-34.

12. Kayman-Kose S, Arioz DT, Toktas H, et al. Transcutaneous electrical nerve stimulation (TENS) for pain control after vaginal delivery and cesarean section. J Matern Fetal Neonatal Med. 2014;27(15):1572-1575.

13. Beleza ACS, Ferreira CHJ, Driusso P, Dos Santos CB, Nakano AMS. Effect of cryotherapy on relief of perineal pain after vaginal childbirth with episiotomy: a randomized and controlled clinical trial. 2017;103(4):453-458. 
14. Pitangui AC, Araújo RC, Bezerra MJ, Ribeiro CO, Nakano AM. Low and high-frequency TENS in post-episiotomy pain relief: a randomized, double-blind clinical trial. Braz J Phys Ther. 2014;18(1):72-8.

15. Pitangui AC, de Sousa L, Gomes FA, Ferreira CH, Nakano AM. High-frequency TENS in post-episiotomy pain relief in primiparous puerpere: a randomized, controlled trial. J Obstet Gynaecol Res. 2012;38(7):980-987.

16. Physical Activity and Exercise During Pregnancy and the Postpartum Period: ACOG Committee Opinion Summary, Number 804 . Obstet Gynecol. 2020 Apr;135(4):991-993.

17. Barakat R, Pelaez M, Lopez C, Montejo R, Coteron J. Exercise during pregnancy reduces the rate of cesarean and instrumental deliveries: results of a randomized controlled trial. J Matern Fetal Neonatal Med 2012;25:2372-6.

18. Nakamura A, van der Waerden J, Melchior M, Bolze C, ElKhoury F, Pryor L. Physical activity during pregnancy and postpartum depression: systematic review and meta-analysis. J Affect Disord 2019;246:29-41.

19. American College of Sports Medicine. Staying Active During Coronavirus Pandemic. Exercise is Medicine. Aug. 4, 2020. Available at: https://www.exerciseismedicine.org/assets/page_documents/EIM_Rx\%20for\%20Health_\%20Staying\%20Active\%20During\%20Coronavirus\%20Pandemic.F Accessed Oct. 18, 2020.

20. Dennis CL, Jackson K, Watson J. Interventions for treating painful nipples among breastfeeding women. Cochrane Database Syst Rev. 2014; (12):CD007366.

21. Patel S, Patel S. The Effectiveness of Lactation Consultants and Lactation Counselors on Breastfeeding Outcomes. J Hum Lact. 2016;32(3):530-541.

22. Liu H, Wang LL, Zhao SJ, Kwak-Kim J, Mor G, Liao AH. Why are pregnant women susceptible to CoVID-19? An immunological viewpoint. J Reprod Immunol. 2020;139:103122.

23. Schwartz DA, Graham AL. Potential Maternal and Infant Outcomes from (Wuhan) Coronavirus 2019-nCoV Infecting Pregnant Women: Lessons from SARS, MERS, and Other Human Coronavirus Infections. Viruses. 2020 Feb 10;12(2):194.

24. Trippella G, Ciarcià M, Ferrari M, Buzzatti C, Maccora I, Azzari C, Dani C, Galli L, Chiappini E. COVID-19 in Pregnant Women and Neonates: A Systematic Review of the Literature with Quality Assessment of the Studies. Pathogens. 2020:18;9(6):485.

25. Collin J, Byström E, Carnahan A, Ahrne M. Public Health Agency of Sweden's Brief Report: Pregnant and postpartum women with severe acute respiratory syndrome coronavirus 2 infection in intensive care in Sweden. Acta Obstet Gynecol Scand. 2020;99(7):819-822.

26. Amorim MMR, Soligo Takemoto ML, Fonseca EBD. Maternal deaths with coronavirus disease 2019: a different outcome from low- to middle-resource countries? Am J Obstet Gynecol. 2020;223(2):298-299.

27. Takemoto MLS, Menezes MO, Andreucci CB, Nakamura-Pereira M, Amorim MMR, Katz L, Knobel R. The tragedy of COVID-19 in Brazil: 124 maternal deaths and counting. Int J Gynaecol Obstet. 2020 Jul 9.

28. Takemoto M, Menezes MO, Andreucci CB, Knobel R, Sousa L, Katz L, Fonseca EB, Nakamura-Pereira M, Magalhães CG, Diniz C, Melo A, Amorim M; Brazilian Group for Studies of COVID-19 and Pregnancy. Clinical characteristics and risk factors for mortality in obstetric patients with severe COVID-19 in Brazil: a surveillance database analysis. 2020 Aug 16:10.1111/1471-0528.16470.

29. Ilington S, Strid P, Tong VT, Woodworth K, Galang RR, Zambrano LD, Nahabedian J, Anderson K, Gilboa SM. Characteristics of Women of Reproductive Age with Laboratory-Confirmed SARS-CoV-2 Infection by Pregnancy Status - United States, January 22-June 7, 2020. MMWR Morb Mortal Wkly Rep. 2020 Jun 26;69(25):769-775.

30. Rasmussen SA, Smulian JC, Lednicky JA, Wen TS, Jamieson DJ. Coronavirus Disease 2019 (COVID-19) and pregnancy: what obstetricians need to know. Am J Obstet Gynecol. 2020 May;222(5):415-426.

31. Karimi-Zarchi M, Neamatzadeh H, Dastgheib SA, Abbasi H, Mirjalili SR, Behforouz A, Ferdosian F, Bahrami R. Vertical Transmission of Coronavirus Disease 19 (COVID-19) from Infected Pregnant Mothers to Neonates: A Review. Fetal Pediatr Pathol. 2020 Jun;39(3):246-250.

32. Centers for Disease Control and Prevention. Interim infection prevention and control recommendations for patients with confirmed 2019 novel coronavirus (2019- nCoV) or patients under investigation for 2019-nCoV in healthcare settings. Centers for Disease Control and Prevention. Updated Jun. 3, 2020. Available at: https:// www.cdc.gov/coronavirus/2019-nCoV/hcp/ infection-control.html. Accessed Oct. 18, 2020.

33. Centers for Disease Control and Prevention. Pregnancy, Breastfeeding, and Caring for Newborns. Update Sep. 11, 2020. Available at: https://www.cdc.gov/coronavirus/2019-ncov/need-extra-precautions/pregnancy-breastfeeding.html. Accessed Oct. 18, 2020.

34. Centers for Disease Control and Prevention. Considerations for Inpatient Obstetric Healthcare Settings. May 2020. Available at: https://www.cdc.gov/coronavirus/2019-ncov/hcp/inpatient-obstetric-healthcare-guidance.html_Accessed Oct. 18, 2020.

35. American College of Obstetricians and Gynecologists (ACOG). Novel Coronavirus 2019 (COVID-19). Practice Advisory. Update Sept. 25, 2020. Available at: https://www.acog.org/clinical/clinical-guidance/practice-advisory/articles/2020/03/novel-coronavirus-2019. Accessed Oct. 18, 2020.

36. World Health Organization.Q\&A: Pregnancy, childbirth and COVID-19. Sept. 2, 2020. Available at: https://www.who.int/emergencies/diseases/novelcoronavirus-2019/question-and-answers-hub/q-a-detail/q-a-on-covid-19-pregnancy-and-childbirth. Accessed Oct. 18, 2020.

37. Centers for Disease Control and Prevention. Using Telehealth to Expand Access to Essential Health Services during the COVID-19 Pandemic. Update Jun 10, 2020. Available at: https://www.cdc.gov/coronavirus/2019-ncov/hcp/telehealth.html. Accessed Oct. 18, 2020.

38. Centers for Disease Control and Prevention. Optimizing Personal Protective Equipment (PPE) Supplies. Update July, 16, 2020. Available at: https://www.cdc.gov/coronavirus/2019-ncov/hcp/ppe-strategy/index.html_.Accessed Oct. 18, 2020.

39. Dantas LO, Barreto RPG, Ferreira CHJ. Digital physical therapy in the COVID-19 pandemic. Braz J Phys Ther. 2020 Sep-0ct;24(5):381-383.

40. Ferreira CHJ, Driusso P, Haddad JM, Pereira SB, Fernandes ACNL, Porto D, Reis BM, Mascarenhas LR, Brito LGO, Ferreira EAG. A guide to physiotherapy in urogynecology for patient care during the COVID-19 pandemic. Int Urogynecol J. 2020 Sep 28:1-8. 
41. Tolcher MC, McKinney JR, Eppes CS, Muigai D, Shamshirsaz A, Guntupalli KK, Nates JL. Prone Positioning for Pregnant Women With Hypoxemia Due to Coronavirus Disease 2019 (COVID-19). Obstet Gynecol. 2020 Aug;136(2):259-261.

42. Samanta S, Samanta S, Wig J, Baronia AK. How safe is the prone position in acute respiratory distress syndrome at late pregnancy? Am J Emerg Med. 2014 Jun;32(6):687.e1-3.

43. Oliveira C, Lopes MAB, Rodrigues AS, Zugaib M, Francisco RPV. Influence of the prone position on a stretcher for pregnant women on maternal and fetal hemodynamic parameters and comfort in pregnancy. Clinics (Sao Paulo). 2017 Jun;72(6):325-332.

44. Felten-Barentsz KM, van Oorsouw R, Klooster E, Koenders N, Driehuis F, Hulzebos EHJ, van der Schaaf M, Hoogeboom TJ, van der Wees PJ. Recommendations for Hospital-Based Physical Therapists Managing Patients With COVID-19. Phys Ther. 2020 Aug 31;100(9):1444-1457.

\section{Supplementary Files}

This is a list of supplementary files associated with this preprint. Click to download.

- Box18.docx 